\title{
Enseñanza-Aprendizaje de la escritura en Educación Infantil y Primaria
}

\author{
Mª José González Valenzuela, Myriam Delgado Ríos \\ Dpto. de Psicología Evolutiva y de la Educación, \\ Universidad de Málaga (España)
}

\section{España}

Ma José González Valenzuela. Universidad de Málaga, Facultad de Psicología. Campus de Teatinos s/n. 29071 Málaga. Spain. E-mail: valenzu@uma.es

(C) Education \& Psychology I+D+i and Editorial EOS (Spain) 


\section{Resumen}

Introducción. El objetivo de este trabajo es analizar los efectos de la aplicación de un programa de intervención psicoeducativa, desde edades tempranas, en el rendimiento en escritura, en términos de copia y dictado. La finalidad de dicho programa es, por un lado, fomentar sistemáticamente el conocimiento fonológico, el desarrollo fonológico, el desarrollo semántico y el morfosintáctico y, por otro lado, priorizar y sistematizar el acto lectoescritor en el curriculum escolar.

Método. La muestra esta formada por 106 alumnos, pertenecientes a zonas socioculturales medio-bajas, con inteligencia normal y sin handicaps físicos, psíquicos y/o sensoriales, que son evaluados desde que comienzan $2^{\circ}$ curso de Educación Infantil hasta que terminan $1^{\circ}$ curso de Educación Primaria. El diseño es de medidas pretest-postests (cuatro evaluaciones) con fases de intervención (tres fases), dos variables de estudio (rendimiento en copia y dictado) y dos grupos de sujetos (control y experimental). Se han calculado los estadísticos descriptivos y se ha realizado análisis de varianza de medidas repetidas.

Resultados. Los resultados obtenidos indican mejores puntuaciones, tanto en copia como en dictado, a lo largo de todas las evaluaciones y un avance significativamente mayor en el grupo entrenado con respecto al de los sujetos no entrenados.

Conclusión. Estos resultados demuestran la eficacia a largo plazo en nuestra lengua de una intervención temprana del lenguaje oral y escrito y la necesidad de realizar cambios en los objetivos curriculares escolares, que mejoren el proceso de enseñanza-aprendizaje de la escritura.

Palabras Clave: Enseñanza-aprendizaje. Rendimiento en copia. Rendimiento en dictado. Educación Infantil y Primaria.

Recepción: 18-07-07 Aceptación Provisional: 08-10-07 Aceptación definitiva: 15-10-07 


\section{Introducción}

La importancia que la escritura tiene en el desarrollo del individuo es reconocida tradicionalmente. La escritura es una competencia básica que se aprende en la escuela a partir de la enseñanza obligatoria y sobre la que se construye el conjunto de conocimientos que conforman los cimientos de lo que ha de ser una formación a lo largo de la vida. Es, además, una estrategia importante para favorecer los aprendizajes de los alumnos.

El análisis del proceso de enseñanza-aprendizaje de la escritura es un tema de interés y de debate abierto en la comunidad científica y profesional debido, entre algunas razones, al hecho de que existen diferentes posturas teóricas y metodológicas en el ámbito de estudio de la intervención psicoeducativa en éste área. El cuándo y el cómo poner en marcha el proceso de enseñanza-aprendizaje de la lengua escrita para conseguir unos resultados óptimos es un tema controvertido y ha sido uno de los motivos que justifica los objetivos de este trabajo.

A este respecto, según la opinión de algunos investigadores, el inicio del proceso de enseñanza-aprendizaje de la escritura debe estar supeditado a la madurez de los sujetos, teniendo sentido que comience cuando los sujetos estén preparados para ello y su maduración neuropsicológica lo permita (a partir de los seis años), que es cuando se conseguiría un adecuado aprendizaje (Condemarín, Chandwick y Milicia, 1985; Downing y Thackray, 1974; Molina, 1991; Muchelli y Bourcier, 1985; Revuelta y Guillen, 1987). Sin embargo, desde otras perspectivas teóricas se defiende que la iniciación de estos aprendizajes no debe estar totalmente supeditada a la madurez del sujeto y se aboga más por una intervención temprana (Baroccio y Hagg, 1999; Blair-Larsen y Williams, 1999; Burns, Griffin y Snows, 1999; Escamilla, 1994; Osborn y Lehr, 1998; Russo, Kosman, Ginsburg, Thompson-Hoffman y Pederson, 1998; Slavin, Madden, Dolan y Wasik, 1996; Swartz, Shook y Klein, 2000).

Por otra parte, cabe destacar que algunos investigadores han considerado la enseñanza-aprendizaje de la escritura desde el desarrollo de prerrequisitos básicos, como la psicomotricidad, percepción visoespacial, lateralidad y/o esquema corporal (Condemarín, y cols. 1985; Downing y Thackray, 1974; Molina, 1991; Muchelli y Bourcier, 1985; Revuelta y Guillen, 1987), frente a otros que defienden que estos no son los prerrequisitos más predictivos del éxito escritor y apuestan más por el fomento del desarrollo de la metacognición y del lenRevista Electrónica de Investigación Psicoeducativa, No 13 Vol 5 (3), 2007. ISSN:1696-2095. pp:651-678 - 653 - 
guaje oral (Adams, 1998; Calero y Pérez, 1993; Carrillo, 1994; Casalis y Louis-Alexandre, 2000; Da Fontoura y Siegel, 1995; Defior y Tudela, 1994; González, 1996; Mahony, Singson y Mann, 2000; Muter y Snowling, 1998; Slavin et al, 1996; So y Siegel, 1997; Stanovich, 1992; Swartz, et al. 2000).

Así pues, desde perspectivas teóricas y metodológicas actuales se defiende la viabilidad y eficacia, en primer lugar, de adelantar la aproximación al lenguaje escrito, iniciando la enseñanza a partir de los tres-cuatro años, para que a partir de los seis años los alumnos puedan acceder a los distintos contenidos curriculares a través de la escritura (Baroccio y Haggs, 1999; Escamilla, 1994; Slavin, 1995; Swartz, et al. 2000). En segundo lugar, abogan por la eficacia de fomentar el desarrollo cognitivo-lingüístico, mejorando el desarrollo fonológico y metafonológico, el desarrollo semántico y morfosintáctico para optimizar el proceso de enseñanza-aprendizaje de la escritura (Adams, 1998; Calero y Perez, 1993; Carrillo, 1994; Casalis y Louis-Alexandre, 2000; Da Fontoura y Siegel, 1995; Defior y Tudela, 1994; Elbro,1996; González, 1993, 1996; Mahony, Singson y Mann, 2000; Seymour y Evans, 1994; Slavin, 1995; Slavin, et al. 1996; So y Siegel, 1997; Swartz, et al. 2000).Y, finalmente, destacan la importancia de priorizar la enseñanza de la lengua escrita y del hábito lectoescritor, a través de la sistematización de distintos modos de lectura y escritura (guiada, compartida, independiente) y del aumento del tiempo de exposición a la lectura y la escritura, como modo de instrucción y de aprendizaje de los contenidos curriculares (Baroccio y Haggs, 1999; Button, Johnson y Ferguson, 1996; Escamilla, 1994; Slavin, 1995; Slavin, Madden, Dolan y Wasik, 1996; Swartz, et al. 2000).

En este sentido, teniendo en cuenta los resultados de distintos trabajos científicos en otras lenguas y en otros sistemas educativos, en este estudio se defiende la importancia de una intervención temprana en el aprendizaje de la escritura, enfatizando el cuándo y el cómo conducir el proceso de enseñanza-aprendizaje desde una perspectiva constructivista y psicolingüística, ya que la optimización del proceso de enseñanza-aprendizaje de la lengua escrita y el uso de la escritura como modo de instrucción podría evitar la aparición de muchos de los atenuantes del fracaso escolar, facilitar el rendimiento académico de los alumnos y mejorar el desarrollo profesional de los maestros. 
A este respecto, el objetivo de este trabajo ${ }^{1}$ es analizar los efectos de la aplicación de un programa de intervención psicoeducativa en el rendimiento en escritura (exactitud en copia y exactitud en dictado) desde Educación Infantil hasta $1^{\circ}$ de Educación Primaria, cuya finalidad es fomentar el desarrollo cognitivo-lingüístico y priorizar y sistematizar la lengua escrita, a través de una propuesta de cambio curricular en el aula.

En función de dichos objetivos se pretende comprobar, que tras la aplicación del programa de intervención, los alumnos del grupo a los que se les aplica el programa de intervención (grupo experimental) alcanzan mejor rendimiento tanto en exactitud en copia como en exactitud en dictado que los alumnos a los que no se les aplique el programa y siguen el currículum oficial ordinario (grupo control).

\section{Método}

\section{Participantes}

La elección de la muestra se llevó a cabo tras distintas actuaciones. En primer lugar, todos los colegios (públicos y concertados) de Málaga capital (España) fueron catalogados en tres grupos, según la zona de alfabetización de la provincia (alta, media y baja). En segundo lugar, se eligieron al azar distintos colegios públicos y concertados de las zonas de alfabetización media y baja, formándose dos grupos, los que aplicarían voluntariamente el programa de intervención (grupo experimental) y los que no aplicarían el programa (grupo control). Finalmente, en cada grupo de estudio se eligieron a aquellos sujetos de habla castellana que no presentaban handicaps físicos, psíquicos o sensoriales y con un nivel intelectual normal. Así, la regla de asignación de los sujetos a cada grupo ha sido no aleatoria y no conocida, que es la que se suele utilizar en este ámbito de estudio (Anguera, Arnau, Ato, Martínez, Pascual y Va1lejo, 1995), ya que los grupos son similares solo en algunas variables no pudiéndose controlar a priori que fuesen iguales en todas las variables de estudio (los sujetos pertenecen a aulas ya formadas en los centros escolares). 
Por tanto, la muestra quedó constituida por un total de 106 sujetos, distribuidos entre el grupo control $(n=32)$ y el grupo experimental $(n=74)$, pertenecientes a diferentes colegios públicos y concertados de zonas socioculturales medio-baja de Málaga capital. Todos los sujetos empezaron cursando $2^{\circ}$ de Educación Infantil, con edades comprendidas entre 3 y 4 años, hasta finalizar $1^{\circ}$ de Educación Primaria con edades comprendidas entre 6 y 7 años, ninguno padecían handicaps físicos, psíquicos o sensoriales y presentaban un nivel intelectual normal.

\section{Instrumentos}

Los instrumentos que se han utilizado para la evaluación de las distintas variables de estudio han sido las pruebas REC y RED, que miden respectivamente rendimiento en exactitud en copia y en exactitud en dictado (Delgado, Martín, Rivas y González, 2004).

Por un lado, la prueba REC está compuesta por cuatro subpruebas: Copia de Figuras, formada por ocho ítems representados por figuras de distinta complejidad gráfica; Copia de Letras, que esta formada por siete ítems, 3 vocales y 4 consonantes; Copia de Palabras, constituida por ocho palabras bisílabas y trisílabas directas y trabadas; y, finalmente, Copia de frases, que esta formada por 2 frases de tres y cinco palabras, respectivamente. Por otro lado, la prueba RED está compuesta por las siguientes subpruebas: Dictado de letras, que está constituida por ocho ítems (2 vocales y 6 consonantes); Dictado de palabras, que esta formada por ocho palabras monosílabas y trisílabas directas y trabadas; Dictado de pseudopalabras, constituida por ocho pseudopalabras bisílabas y trisílabas directas trabadas e inversas; Dictado de frases, que esta compuesta por cuatro ítems que incluyen frases de tres, cuatro y hasta cinco palabras de distinta estructura lingüística (bisílabas directas e inversas, polisílabas y palabras con sílabas trabadas); y, por último, la subprueba Dictado de textos, que está formada por tres textos graduados de menor a mayor longitud y dificultad lingüística. 


\section{Diseño y procedimiento}

El diseño de estudio es cuasi-experimental y longitudinal, con medidas pretest-postest (cuatro mediciones) y fases de intervención (tres fases), con un grupo experimental (al cual se le aplica el programa de intervención psicoeducativa) y un grupo control (que sigue el curriculum oficial establecido). Las variables de estudio son rendimiento en exactitud en copia (REC) y el rendimiento en exactitud en dictado (RED).

El procedimiento llevado a cabo comprende distintas fases a lo largo de tres cursos académicos. La primera fase, se trata de una evaluación inicial (pretest) realizada en Septiembre, al inicio del primer curso académico, con la que se pretende medir la línea base de los alumnos de cuatro años pertenecientes a $2^{\circ}$ curso de Educación Infantil. La segunda fase, corresponde al primer periodo de intervención (Octubre-Mayo del mismo curso académico), en el que se empieza a aplicar el programa elaborado para los niños de $2^{\circ}$ de Educación Infantil (4 años) del grupo experimental. La tercera fase, hace referencia a una fase de evaluación postest ( $2^{\circ}$ evaluación) realizada en Junio, en la que se evalúan nuevamente a los niños (4 años), con el fin de comprobar qué efectos ha tenido en ellos el programa de intervención en esas edades y variables de estudio en comparación con los del grupo control. La cuarta fase, se corresponde con el segundo periodo de intervención (Octubre-Mayo del siguiente curso académico), en la que se continua con la aplicación del programa de intervención elaborado para el grupo experimental que cursan ya $3^{\circ}$ de Educación Infantil ( 5 años). La quinta fase, hace referencia a otra fase de evaluación postest ( $3^{\circ}$ evaluación) realizada en Junio, en la que se evalúan nuevamente a los niños (con 5 años) con el fin de comprobar los efectos del periodo de intervención, en las diferentes variables de estudio en comparación con los niños del grupo control. La sexta fase, hace referencia al $3^{\circ}$ periodo de intervención, (Octubre-Mayo del siguiente curso académico) en la que se termina con la aplicación del programa elaborado para los niños del grupo experimental, que cursan ya $1^{\text {o }}$ de Educación Primaria (6 años). Y por último, la séptima fase (Junio del mismo curso académico) se corresponde con la última fase de evaluación ( $4^{\circ}$ evaluación), en la que se evalúan nuevamente a los niños (con 6 años) con las mismas pruebas que en las evaluaciones anteriores, con el fin de comprobar qué efectos ha tenido finalmente la aplicación del programa en las diferentes variables de estudio en comparación con el grupo control, a lo largo de los tres cursos académicos. 
Las evaluaciones llevadas a cabo se realizan a cada sujeto individualmente de forma ciega, en horario lectivo y en un aula acondicionada para tal fin, con las pruebas descritas anteriormente.

En las fases de intervención, los sujetos del grupo control no recibieron la aplicación del programa de intervención diseñado sino que recibieron la enseñanza reglada por los objetivos curriculares oficiales de Educación Infantil y Primaria, establecidos por la Consejería de Educación y Cultura de la Junta de Andalucía (Decreto 105 y 107, 1992). Cabe destacar, por un lado, que las maestras que llevaron a cabo la enseñanza de estos contenidos en las aulas ordinarias no recibieron ningún tipo de información o preparación previa sobre la enseñanza de la lengua escrita, y por otro lado, a pesar de que estos contenidos estaban relacionados con la aproximación al lenguaje escrito y con el fomento del lenguaje oral en Educación Infantil no se desarrollaron de forma sistemática y prioritaria en el aula, dedicándoles una duración semanal no concreta y no constante. Además, el inicio de la enseñanza de la lectura de manera más específica se llevó a cabo a partir del primer curso de Educación Primaria.

En cuanto a los sujetos del grupo experimental, se les aplicó el programa de intervención elaborado conjuntamente con las maestras que lo aplicaron en las aulas, realizándose seminarios y talleres donde se establecieron conjuntamente los objetivos y contenidos del mismo, creándose y recopilándose materiales necesarios para su aplicación. Estos seminarios y talleres también sirvieron para la instrucción y el adiestramiento de las maestras en cuanto al análisis de los procesos psicológicos implicados en la lengua escrita y de los métodos de enseñanza de la misma, recomendándoles lecturas de libros y artículos sobre estos contenidos. La aplicación del programa de intervención se lleva a cabo por las maestras en las aulas ordinarias y se realiza un seguimiento periódico, a través de entrevistas y talleres con ellas, individualmente y en grupo, con el fin de analizar sus experiencias, resolver las dificultades que se iban encontrando y comentar y valorar los logros obtenidos.

Los objetivos establecidos en el programa de intervención psicoeducativa que se le aplicaba al grupo experimental hacen referencia, por un lado, al fomento sistemático en el aula del acto lector y escritor, ya que una manera de conseguir eficacia en estos aprendizajes 
es a través del contacto directo y continuo con ellos (Slavin, 1995; Stanovich, 1994; Swartz, 1996). Por otro lado, al fomento sistemático del conocimiento fonológico (conocimiento silábico y fonémico), del desarrollo fonológico (articulación y discriminación de fonemas), del desarrollo semántico (categorización de conceptos e identificación de familias léxicas) y del desarrollo morfosintáctico (identificación y distinción del valor de las palabras en distintos tipos de oraciones), como han propuesto diferentes trabajos en esta línea (Carrillo, 1994; Casalis y Louis-Alexandre, 2000; Da Fontoura y Siegel, 1995; Defior y Tudela, 1994; Gillon y Dodd, 1994; González, 1993, 1996; So y Siegel, 1997).

Para el desarrollo de estos objetivos se elaboraron una serie de contenidos a trabajar por trimestre y por año de intervención, organizados secuencialmente, según criterios evolutivos y educativos, que se exponen en la tabla 1. Dichos contenidos (lectoescritura, conocimiento fonológico y desarrollo del lenguaje oral) se trabajan de manera conjunta e interrelacionada, de forma que la enseñanza-aprendizaje de unos refuerza la de los otros.

Tabla 1. Contenidos secuenciados de Programa de intervención psicoeducativa

\begin{tabular}{|c|c|}
\hline AÑO & LECTOESCRITURA \\
\hline $\mathbf{1}^{\mathbf{o}}$ & $\begin{array}{l}\text { Reconocimiento de imágenes y pictogramas } \\
\text { - Reconocimiento de vocales y consonantes } p, m \\
\text { - Reconocimiento de las letras } n, \tilde{n}, l, s, t, j \\
\text { - Reconocimiento de las letras } d, b, c \text {, } \\
\text { - Reconocimiento de sílabas directas con esas letras } \\
\text { - Reconocimiento de palabras bisílabas con esas letras } \\
\text { - Reconocimiento de frases cortas (2-3 palabras) con esas palabras } \\
\text { - Reconocimiento de cuento corto ( } 2 \text { cuentos) } \\
\text { - Comprensión de palabras y frases leídas con esas letras } \\
\text { - Comprensión de cuento corto con las palabras y frases trabajadas }\end{array}$ \\
\hline $2^{\circ}$ & $\begin{array}{l}\text { Reconocimiento de pictogramas } \\
\text { - Repaso de letras: vocales, } \mathrm{p}, \mathrm{m}, \mathrm{n}, \tilde{\mathrm{n}}, \mathrm{l} \\
\text { - Reconocimiento de letras: } \mathrm{s}, \mathrm{t}, \mathrm{j}, \mathrm{d}, \mathrm{y}, \mathrm{b} / \mathrm{v}, \mathrm{z} / \mathrm{c} \\
\text { - Reconocimiento de letras: } \mathrm{r}, \mathrm{f}, \mathrm{h}, \mathrm{ch}, \mathrm{k}, \mathrm{c} / \mathrm{q}, \mathrm{g}, \mathrm{x}, \mathrm{w} \\
\text { - Reconocimiento de sílabas directas con esas letras } \\
\text { - Reconocimiento de sílabas inversas con } \mathrm{s}, \mathrm{l}, \mathrm{n} \\
\text { - Reconocimiento de sílabas inversas con } \mathrm{r}, \mathrm{j}, \mathrm{z}, \mathrm{m}, \mathrm{p}, \mathrm{b}, \mathrm{d}, \mathrm{t}, \mathrm{c}, \mathrm{g}, \mathrm{f} \\
\text { - Reconocimiento de sílabas directas dobles: pr/pl, br/bl, tr, dr, cr/cl, fr/fl, gl/gr } \\
\text { - Reconocimiento de palabras cortas familiares y no familiares (bisílabas y trisílabas) } \\
\text { - Reconocimiento de frases cortas (2-3 palabras) (4-6 palabras) y (6-8 palabras) } \\
\text { - Reconocimiento de cuentos cortos (2 cuentos) en cada trimestre } \\
\text { - Comprensión lectora de palabras cortas familiares (bisílabas y trisílabas) y no familiares } \\
\text { - Comprensión lectora de frases de } 3,4-6 \text { y 6-8 palabras } \\
\text { - Comprensión lectora de cuentos cortos: ideas principales } \\
\text { - Redacción dirigida con dibujos sencillos (descripción escrita) }\end{array}$ \\
\hline
\end{tabular}




\begin{tabular}{|c|c|}
\hline & -Redacción no dirigida (descripción escrita) \\
\hline $3^{\mathbf{0}}$ & $\begin{array}{l}\text { Reconocimiento de todas las letras en mayúsculas y minúsculas } \\
\text { - Reconocimiento de todas las sílabas directas, inversas y mixtas } \\
\text { - Reconocimiento de palabras cortas y largas conocidas y desconocidas } \\
\text { - Reconocimiento de frases (6 palabras) (8 palabras) (10 palabras) } \\
\text { - Reconocimiento de texto ( } 3 / 4,4 / 5 \text { y } 5 / 6 \text { frases) } \\
\text { - Reconocimiento de cuentos ( } 4 \text { cuentos) en cada trimestre } \\
\text { - Comprensión lectora de palabras poco frecuente (bisílabas y trisílabas) } \\
\text { - Comprensión de frases (de } 8 \text { a } 10 \text { palabras) } \\
\text { - Comprensión de textos } \\
\text { - Preguntas orales sobre aspectos explícitos en los textos narrativos: personajes, desarrollo y desenla- } \\
\text { ce } \\
\text { - Preguntas escritas sobre ideas principales explícitas e implícitas en los textos narrativos: personajes, } \\
\text { desarrollo, desenlace } \\
\text { - Preguntar a los niños sobre el texto según el título } \\
\text { - Resumen oral de lo leído } \\
\text { - Cambiar oralmente el final de un cuento o texto } \\
\text { - Composición de escritos (organización de ideas y ortografía) } \\
\text { - Redacción dirigida con dibujos } \\
\text { - Redacción dirigida con palabras y/o frases dadas (comienzo y final) } \\
\text { - Redacción espontánea con organización de ideas: describir un objeto o persona } \\
\text { - Redacción espontánea con organización de ideas: describir un suceso o acontecimiento } \\
\text { Redacción de un cuento en grupo }\end{array}$ \\
\hline
\end{tabular}

Tabla 1. Contenidos secuenciados de Programa de intervención psicoeducativa (continuación).

\begin{tabular}{|c|c|c|c|c|}
\hline A $\tilde{N} O$ & \begin{tabular}{|c|} 
CONOCIMIENTO \\
FONOLÓGICO
\end{tabular} & $\begin{array}{l}\text { DESARROLLO } \\
\text { FONOLÓGICO }\end{array}$ & \begin{tabular}{|c|} 
DESARROLLO \\
SEMÁNTICO
\end{tabular} & $\begin{array}{c}\text { DESARROLLO } \\
\text { MORFOSINTÁCTICO }\end{array}$ \\
\hline $1^{0}$ & $\begin{array}{l}\text {-Contar sílabas en pala- } \\
\text { bras con las letras } \\
\text { trabajadas } \\
\text {-Identificar sílabas inicia- } \\
\text { les con las letras } \\
\text { trabajadas } \\
\text {-Identificar rimas } \\
\text { - Identificación de fone- } \\
\text { mas en posición inicial } \\
\text {-Identificación de fone- } \\
\text { mas en cualquier posi- } \\
\text { ción } \\
\text {-Reconocimiento estruc- } \\
\text { tura vocálica de palabras } \\
\text { con las letras trabajadas }\end{array}$ & $\begin{array}{l}\text {-Articulación y } \\
\text { discriminación de } \\
\text { los sonidos (fo- } \\
\text { nemas) } \\
\text { de las letras estu- } \\
\text { diadas. }\end{array}$ & $\begin{array}{l}\text {-Definir dibujos (perso- } \\
\text { nas, objetos, accio- } \\
\text { nes...) } \\
\text {-Elegir el término que } \\
\text { define a un dibu- } \\
\text { jo(personas, objetos, } \\
\text { acciones...) } \\
\text {-Asociar dibujo-palabra } \\
\text { y definirla. } \\
\text {-Clasificar dibujos de } \\
\text { distintas categorías } \\
\text { (animales, ropas, ju- } \\
\text { guetes...) }\end{array}$ & $\begin{array}{l}\text { Ordenar } 2 / 3 \text { dibujos para } \\
\text { construir una historia y } \\
\text { explicarla } \\
\text {-Ordenar } 2 / 3 \text { palabras para } \\
\text { construir una frase con las } \\
\text { palabras trabajadas } \\
\text {-Completar frases }(2 / 3 \\
\text { palabras) ofreciéndoles } \\
\text { alternativas }\end{array}$ \\
\hline $2^{\circ}$ & $\begin{array}{l}\text {-Contar sílabas en pala- } \\
\text { bras con las letras traba- } \\
\text { jadas } \\
\text {-Identificar sílabas en } \\
\text { cualquier posición } \\
\text {-Identificar rimas } \\
\text {-Añadir sílabas } \\
\text {-Omitir sílabas } \\
\text {-Encadenamiento de } \\
\text { palabras } \\
\text {-Identificación de fonemas } \\
\text { en cualquier posición }\end{array}$ & $\begin{array}{l}\text {-Articulación y } \\
\text { discriminación de } \\
\text { los sonidos (fo- } \\
\text { nemas)de las le- } \\
\text { tras estudiadas. }\end{array}$ & $\begin{array}{l}\text {-Definir dibujos (perso- } \\
\text { nas, objetos, acciones) } \\
\text {-Definición oral de } \\
\text { palabras } \\
\text {-Asociación dibujo- } \\
\text { palabra } \\
\text {-Asociar dibujo- } \\
\text { palabra-definición } \\
\text {-Asociación palabra- } \\
\text { definición } \\
\text {-Clasificar dibujos por } \\
\text { categoría semántica } \\
\end{array}$ & $\begin{array}{l}\text {-Contar palabras de una } \\
\text { frase oral y escrita } \\
\text {-Ordenar dibujos para } \\
\text { construir una historia oral } \\
\text {-Completar frases con } \\
\text { dibujos } \\
\text {-Ordenar 3-4//4-5 palabras } \\
\text { para construir una frase } \\
\text {-Completar frases con } \\
\text { palabras } \\
\text { alternativas de apoyo y sin } \\
\text { apoyo }\end{array}$ \\
\hline
\end{tabular}




\begin{tabular}{|c|c|c|c|c|}
\hline & $\begin{array}{l}\text {-Reconocimiento de la } \\
\text { estructura vocálica de las } \\
\text { palabras } \\
\text {-Recuento de fonemas en } \\
\text { palabras } \\
\text {-Adición de fonemas en } \\
\text { palabras } \\
\text {-Omisión de fonemas en } \\
\text { palabras }\end{array}$ & & & \\
\hline $3^{\circ}$ & $\begin{array}{l}\text {-Identificar y Hacer rimas } \\
\text {-Añadir sílabas } \\
\text {-Omitir sílabas } \\
\text {-Encadenamiento de } \\
\text { palabras por las sílabas } \\
\text { finales } \\
\text {-Sustituir sílabas } \\
\text {-Añadir fonemas } \\
\text {-Omitir fonemas } \\
\text {-Encadenamiento de } \\
\text { palabras por los fonemas } \\
\text { finales } \\
\text {-Deletrear monosílabas, } \\
\text { bisílabas y trisílabas } \\
\text { directas, inversas y mix- } \\
\text { tas } \\
\text {-Sustituir fonemas } \\
\text {-Adivinar deletreo de } \\
\text { monosílabas y bisílabas } \\
\text { directas e inversas }\end{array}$ & $\begin{array}{l}\text {-Articulación y } \\
\text { discriminación de } \\
\text { los sonidos (fo- } \\
\text { nemas) de las } \\
\text { letras estudiadas. }\end{array}$ & $\begin{array}{l}\text {-Categorizar y clasificar } \\
\text { conceptos, según va- } \\
\text { rios criterios } \\
\text {-Identificar absurdos de } \\
\text { contenido } \\
\text {-Buscar sinónimos } \\
\text {-Buscar antónimos } \\
\text {-Construir y resolver } \\
\text { sopa de letras con } \\
\text { definiciones } \\
\text {-Resolver crucigramas }\end{array}$ & $\begin{array}{l}\text {-Completar frases } \\
\text {-Ordenar palabras y cons- } \\
\text { truir frases } \\
\text {-Ordenar frases y construir } \\
\text { un texto } \\
\text {-Concordancia de género } \\
\text { (masculino y femenino) } \\
\text {-Concordancia de número } \\
\text { (singular y plural) }\end{array}$ \\
\hline
\end{tabular}

Finalmente, en cuanto a los procedimientos de aplicación del programa de intervención, cabe destacar que éste llevó a cabo por las maestras en las aulas ordinarias. La dedicación al programa de intervención fue diaria (dos-tres horas) de forma individual y colectiva en todas las materias escolares, con el fin de sistematizar y diversificar lo aprendido y de fomentar la conciencia y el gusto por leer y escribir. Las mayoría de las actividades indicadas se llevaron a cabo a través de ejercicios de lápiz-papel con dibujos atractivos, de la lectura de libros con temas de la vida cotidiana que fomentaban la educación en valores (convivencia, solidaridad, medio ambiente, etc) y cercanos al vocabulario de los niños, dibujando sobre lo leído y escrito y con diálogos y conversaciones donde se formulaban preguntas para que los sujetos aprendieran, reflexionaran y pensaran sobre lo que tenían que realizar y lo que habían llevado a cabo en cada una de las actividades programadas. 


\section{Resultados}

El análisis de los datos realizado ha consistido, por un lado, en el calculo de los estadísticos descriptivos de todas las variables de estudio, y por otro lado, el Análisis de Varianza de Medidas Repetidas con la prueba Anova de dos factores con medidas repetidas en un solo factor, tras comprobar los supuestos de homocedasticidad, con la prueba de Levene, y el supuesto de esfericidad, con la prueba de Mauchly. Se realizaron los diferentes contrastes de hipótesis referidos, por un lado, al factor inter-sujeto y, por otro lado, al factor intra-sujeto, para comprobar si el efecto de la intervención había sido significativo; es decir, para averiguar si las diferencias entre el grupo experimental y el grupo habían sido o no significativas y si existía también diferencias significativas entre las distintas evaluaciones realizadas debido a la intervención. Por último, se realizaron las pruebas post hoc mediante el método basado en la distribución T-Student y en la desigualdad de Bonferroni (Método Bonferroni), que muestra las diferencias específicas entre evaluaciones en cada grupo y las diferencias concretas entre grupos en cada evaluación (Blanca y Rando, 1999).

\section{Resultados obtenidos en Rendimiento en Exactitud en Copia}

En la tabla 2 se presentan los estadísticos descriptivos del Rendimiento en Exactitud en Copia (REC), obtenidos por los dos grupos de sujetos en las cuatro evaluaciones. En esta tabla se observa como ha habido un aumento en las puntuaciones medias en la segunda, tercera y cuarta evaluación con respecto a la evaluación inicial (pretest), tanto en el grupo experimental como en el grupo control (Tabla 2 y Figura 1). Se observa que el grupo experimental ha pasado de tener una $\bar{x}=7.31$ en la evaluación inicial, a una $\bar{x}=19.96$ en la segunda, una $\bar{x}=29.15$ en la tercera, y una $\bar{x}=36.81$ en la cuarta y última evaluación. Por otro lado, también se encuentra como el grupo control ha pasado de tener una $\bar{x}=4.44$ en la evaluación inicial, a una $\bar{x}=8.84, \bar{x}=19.91, y \bar{x}=28.81$ en la segunda, tercera y cuarta evaluación, respectivamente. 
Tabla 2. Estadísticos descriptivos del Rendimiento en Copia y Dictado en las distintas evaluaciones y grupos.

\begin{tabular}{|c|c|c|c|c|c|c|c|c|c|}
\hline \multirow[b]{2}{*}{ Variable } & \multirow[b]{2}{*}{ Grupo } & \multicolumn{2}{|c|}{ Ev.1 } & \multicolumn{2}{|c|}{$E v .2$} & \multicolumn{2}{|c|}{$E v .3$} & \multicolumn{2}{|c|}{$E v .4$} \\
\hline & & $\bar{x}$ & $S_{x}$ & $\bar{x}$ & $S_{x}$ & $\bar{x}$ & $S_{x}$ & $\bar{x}$ & $S_{x}$ \\
\hline \multirow{2}{*}{ REC } & $E$ & 7.31 & 5.65 & 19.96 & 7.66 & 29.15 & 5.33 & 36.81 & 2.26 \\
\hline & $\mathrm{C}$ & 4.44 & 4.92 & 8.84 & 6.21 & 19.91 & 9.11 & 28.81 & 4.73 \\
\hline \multirow[t]{2}{*}{ RED } & $E$ & 0.08 & 0.36 & 2.58 & 1.89 & 9.19 & 17.24 & 91.69 & 27.30 \\
\hline & $\mathrm{C}$ & 0.03 & 0.18 & 0.94 & 1.13 & 5.16 & 6.82 & 42.47 & 28.98 \\
\hline
\end{tabular}

Para analizar si estas diferencias son significativas y debidas al efecto de la intervención se llevó a cabo el análisis de varianza de medidas repetidas en esta variable de estudio, comprobándose el supuesto de Homocedasticidad $\left(F_{(1,104)}==1.85\right.$ y p=0.17) y verificándose el supuesto de Esfericidad $(\varepsilon=0.98)$.

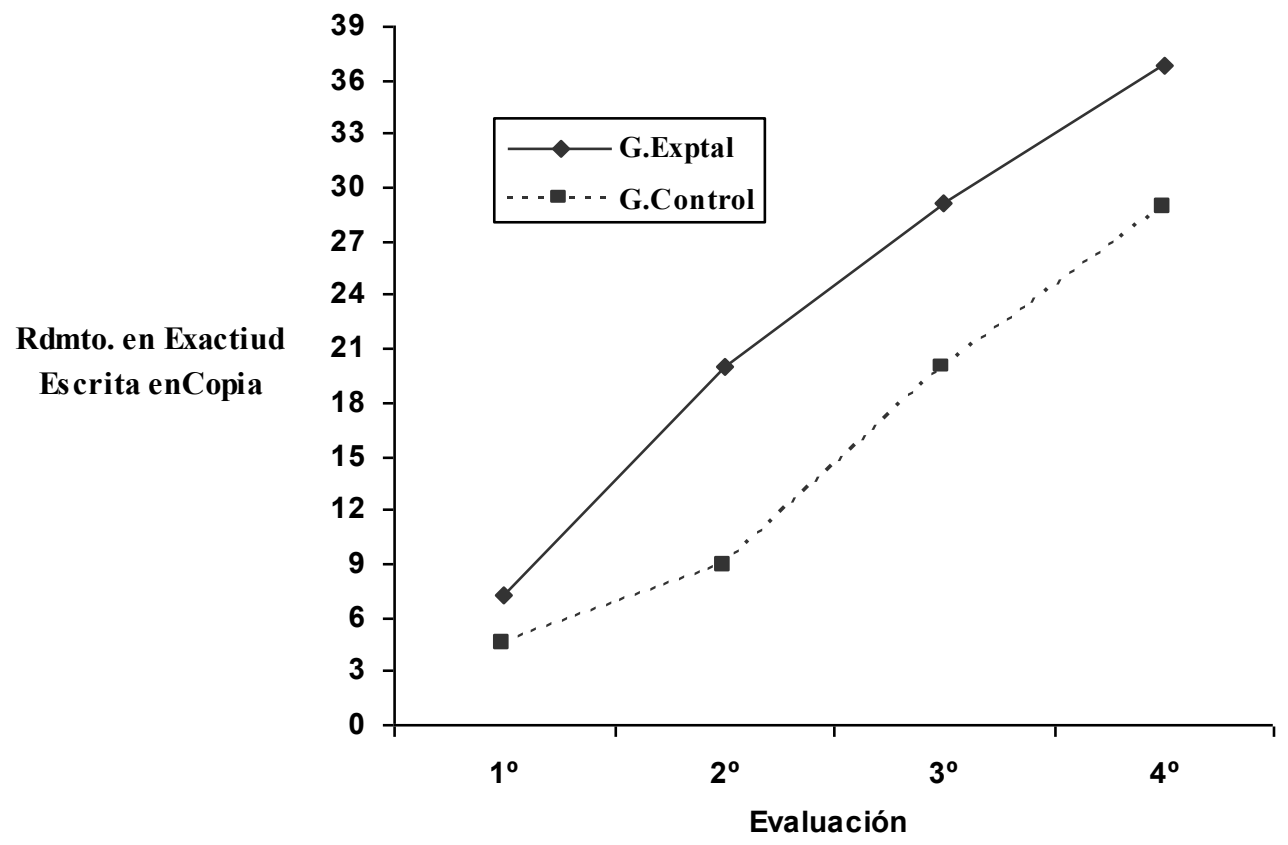

Figura 1. Puntuaciones medias obtenidas en el Rendimiento en Exactitud en Copia. 
El análisis de varianza de medidas repetidas muestra que existen diferencias estadísticamente significativas entre los grupos control y experimental $\left(F_{(1,104)}=78.55 ; \mathrm{p}<0.001\right)$, rechazándose la hipótesis nula del factor inter-sujeto que hace referencia a la no existencia de diferencias entre las medias de los grupos en rendimiento en exactitud en copia. Así mismo, hay que resaltar en este factor la alta adecuación del contraste de hipótesis, obteniéndose una excelente potencia de ( $\beta=1$ ) y un considerado tamaño del efecto, con $\eta^{2}=0.43$ (Tabla 3 ).

Por otro lado, los resultados rechazan la hipótesis nula del factor intra-sujetos, referente a que no existen diferencias significativas entre las diversas evaluaciones, ya que se muestra un cambio significativo a lo largo de las cuatro evaluaciones $\left(F_{\left(2^{\prime} 93,305^{\prime} 69\right)}=544.48\right.$; $\mathrm{p}<0.001)$. Se resalta, la excelente potencia de contraste de hipótesis $(\beta=1)$ en ambos grupos y un considerado tamaño del efecto $\left(\eta^{2}=0.84\right)$ (Tabla 3$)$.

Tabla 3. Análisis de Varianza de Medidas Repetidas en Rendimiento en Copia y Dictado.

\begin{tabular}{lrcccc}
\hline Fuente & mc & gl & F & $\beta$ & $\mathbf{n}^{2}$ \\
\hline REC & \multicolumn{5}{c}{ Inter sujetos } \\
& \multicolumn{7}{c}{. } \\
Grupo (G) & 5446.93 & 1 & $78.55^{* *}$ & 1 & .43 \\
error & 69.33 & 104 & & \\
\hline
\end{tabular}

Intra sujetos

\begin{tabular}{lrrrrr} 
Eval (E) & 12588.58 & 2.93 & $544.48^{* *}$ & 1 & .84 \\
G x E & 284.13 & 2.93 & $12.28^{* *}$ & 1 & .10 \\
error & 23.12 & 305.69 & & & \\
\hline RED & \multicolumn{5}{c}{ Inter sujetos } \\
\multicolumn{7}{c}{} \\
$\quad \begin{array}{c}\text { Grupo (G) } \\
\text { error }\end{array}$ & 23557.61 & 1 & $70.73^{* *}$ & 1 & .40 \\
\hline
\end{tabular}




\begin{tabular}{lrrrrr}
\multicolumn{5}{c}{ Intra sujetos } \\
Eval (E) & 176448.09 & 1.52 & $403.55^{* *}$ & 1 & .79 \\
G x E & 22991.84 & 1.52 & $52.58^{* *}$ & 1 & .33 \\
error & 437.24 & 158.42 & & & \\
\hline $\mathrm{p}<.001$ & & &
\end{tabular}

Así mismo, se encuentra un efecto significativo de interacción evaluación y grupo $\left(F_{\left(2^{\prime} 93,305^{\prime} 69\right)}=12.28 ; \mathrm{p}<0.001\right)$, rechazándose así la hipótesis nula que hace referencia a la no existencia de diferencias significativas entre el grupo experimental y el grupo control en las diversas evaluaciones. Se observa una máxima potencia $(\beta=1)$ y un considerado tamaño del efecto, con $\eta^{2}=0.10$, como se puede observar en la tabla 3 . En este sentido, se puede afirmar que hay diferencias entre los grupos a lo largo de las distintas evaluaciones, a favor del grupo experimental, debida a la aplicación del programa de intervención.

Los contrastes de medida intra-sujetos muestran significativo el efecto lineal, para las evaluaciones $\left(F_{(1,104)}=1763.14 ; \mathrm{p}<0.001\right)$ y el efecto lineal, cuadrático y cúbico también resulta significativo para la interacción $\left(F_{(1,104)}=9.71,17.76\right.$ y $6.89 ; \mathrm{p}<0.001$, respectivamente). Por tanto, puede concluirse que el modelo más plausible para explicar la variabilidad del Rendimiento en Exactitud en Copia, es el lineal, recogiendo los efectos de la evaluación y de su interacción con el grupo.

Por otra parte, tras examinar las diferencias post-hoc mediante el método de Bonferroni, para los niveles de interacción entre cada una de las evaluaciones en cada uno de los grupos, los resultados muestran que existen diferencias estadísticamente significativas entre las diferentes evaluaciones tanto en el grupo experimental como en el grupo control (Tabla 4). Es decir, que tanto en el grupo control como en el grupo experimental, se observan diferencias significativas entre los cuatro y los cinco años, entre los cuatro y los seis años y entre los cinco y los seis años. 
Tabla 4. Comparaciones entre evaluaciones en cada grupo en REC Y RED

\begin{tabular}{|c|c|c|c|c|}
\hline Grupo & $\begin{array}{r}\text { (I) } \\
\text { Evalua }\end{array}$ & $\begin{array}{c}\text { (J) } \\
\text { Evaluación }\end{array}$ & $\begin{array}{c}\text { Diferencia entre } \\
\text { medias } \\
(\mathrm{I}-\mathrm{J})\end{array}$ & Significación $^{\mathrm{a}}$ \\
\hline \multicolumn{5}{|c|}{ REC } \\
\hline \multirow{6}{*}{ Exptal. } & & 2 & $-12,649$ & $*$ \\
\hline & 1 & 3 & $-21,838$ & $*$ \\
\hline & & 4 & $-29,500$ & $*$ \\
\hline & & 3 & $-9,189$ & $*$ \\
\hline & 2 & 4 & $-16,851$ & $*$ \\
\hline & 3 & 4 & $-7,662$ & $*$ \\
\hline \multirow{6}{*}{ Control } & & 2 & $-4,406$ & $*$ \\
\hline & 1 & 3 & $-15,469$ & $*$ \\
\hline & & 4 & $-24,375$ & $*$ \\
\hline & & 3 & $-11,062$ & * \\
\hline & 2 & 4 & $-19,969$ & $*$ \\
\hline & 3 & 4 & $-8,906$ & $*$ \\
\hline \multicolumn{5}{|c|}{ RED } \\
\hline \multirow{6}{*}{ Exptal. } & & 2 & $-2,500$ & $*$ \\
\hline & 1 & 3 & $-19,108$ & $*$ \\
\hline & & 4 & $-91,608$ & $*$ \\
\hline & & 3 & $-16,608$ & $*$ \\
\hline & 2 & 4 & $-89,108$ & $*$ \\
\hline & 3 & 4 & $-72,500$ & $*$ \\
\hline \multirow{6}{*}{ Control } & & 2 &,- 906 & \\
\hline & 1 & 3 & $-5,125$ & \\
\hline & & 4 & $-42,437$ & $*$ \\
\hline & & 3 & $-4,219$ & \\
\hline & 2 & 4 & $-41,531$ & $*$ \\
\hline & 3 & 4 & $-37,312$ & $*$ \\
\hline
\end{tabular}

${ }^{a}$ Ajuste para comparaciones múltiples: Bonferroni $* \mathrm{p}<.05$ 
Por último, las diferencias post-hoc encontradas mediante la prueba $\mathrm{T}$ y la corrección de Bonferroni para los niveles de interacción entre cada uno de los grupos en cada evaluación, nos muestran que existen diferencias estadísticamente significativas en todas las evaluaciones a favor del grupo experimental, encontrándose que las diferencias entre los grupos aumentan a partir de la evaluación inicial, manteniéndose estas hasta la última evaluación (Tabla 5). Es decir, se encontraron diferencias estadísticamente significativas entre ambos grupos a partir de la primera evaluación, a favor del grupo experimental, manteniéndose estas diferencias a lo largo de los diferentes periodos de intervención. De esta manera, como se puede comprobar en la tabla 5, el número de periodos de intervención que han recibido los sujetos, así como el grupo al que pertenecen, explican las diferencias observadas en el Rendimiento en Exactitud en Copia. Es decir, que no sólo se encuentra que el grupo experimental obtiene un mejor Rendimiento en Exactitud en Copia que el grupo control, sino que, además, las diferencias encontradas entre ambos grupos en dicho rendimiento, se mantienen a medida que se avanza en los periodos de intervención que reciben los sujetos del primer grupo (GE).

Tabla 5. Comparaciones por pares entre grupos en cada evaluación en REC y en RED.

\begin{tabular}{|c|c|c|c|c|}
\hline Evaluación & $\begin{array}{c}\bar{x} \\
\text { G. Exptal } \\
(\mathrm{I})\end{array}$ & $\begin{array}{c}\bar{x} \\
\text { G. Control } \\
\text { (J) }\end{array}$ & $\begin{array}{c}\text { Diferencia } \\
\text { entre medias } \\
\quad(\mathrm{I}-\mathrm{J})\end{array}$ & Significación \\
\hline \multicolumn{5}{|c|}{ REC } \\
\hline 1 & 7.31 & 4.44 & 2.87 & $*$ \\
\hline 2 & 19.96 & 8.84 & 11.11 & $*$ \\
\hline 3 & 29.15 & 19.91 & 9.24 & $*$ \\
\hline 4 & 36.81 & 28.81 & 7.99 & $*$ \\
\hline \multicolumn{5}{|c|}{ RED } \\
\hline 1 & 0.08 & 0.03 & 0.04 & \\
\hline 2 & 2.58 & 0.94 & 1.64 & $*$ \\
\hline 3 & 19.19 & 5.16 & 14.03 & $*$ \\
\hline 4 & 91.69 & 42.47 & 49.22 & $*$ \\
\hline
\end{tabular}

${ }^{a}$ Ajuste para comparaciones múltiples: Bonferroni $\mathrm{p}<.05$ 
Por tanto, podemos concluir que los dos grupos avanzan en el transcurso de los diferentes niveles educativos, pero el grupo al que se le aplicó el programa de intervención psicoeducativa ha obtenido un mayor Rendimiento en Exactitud en Copia que el grupo al que no se le aplicó; y que, además, estas diferencias se mantienen a lo largo de los distintos niveles educativos.

\section{Resultados obtenidos en Rendimiento en Exactitud en Dictado}

En la tabla 2 se presentan los estadísticos descriptivos del rendimiento en exactitud en dictado (RED), obtenidos por los dos grupos de sujetos en las cuatro evaluaciones. En esta tabla se observa como ha habido un aumento en las puntuaciones medias en la segunda, tercera y cuarta evaluación con respecto a la evaluación inicial (pretest), tanto en el grupo experimental como en el grupo control (Tabla 2 y Figura 2). Se observa que el grupo experimental ha pasado de tener una $\bar{x}=0.08$ en la evaluación inicial, a una $\bar{x}=2.58$ en la segunda, una $\bar{x}=9.19$ en la tercera, y una $\bar{x}=91.69$ en la cuarta y última evaluación. Por otro lado, también se encuentra como el grupo control ha pasado de tener una $\bar{x}=0.03$ en la evaluación inicial, a una $\bar{x}=0.94 ; \bar{x}=5.16, \mathrm{y} \quad \bar{x}=42.47$ en la segunda, tercera y cuarta evaluación, respectivamente. 


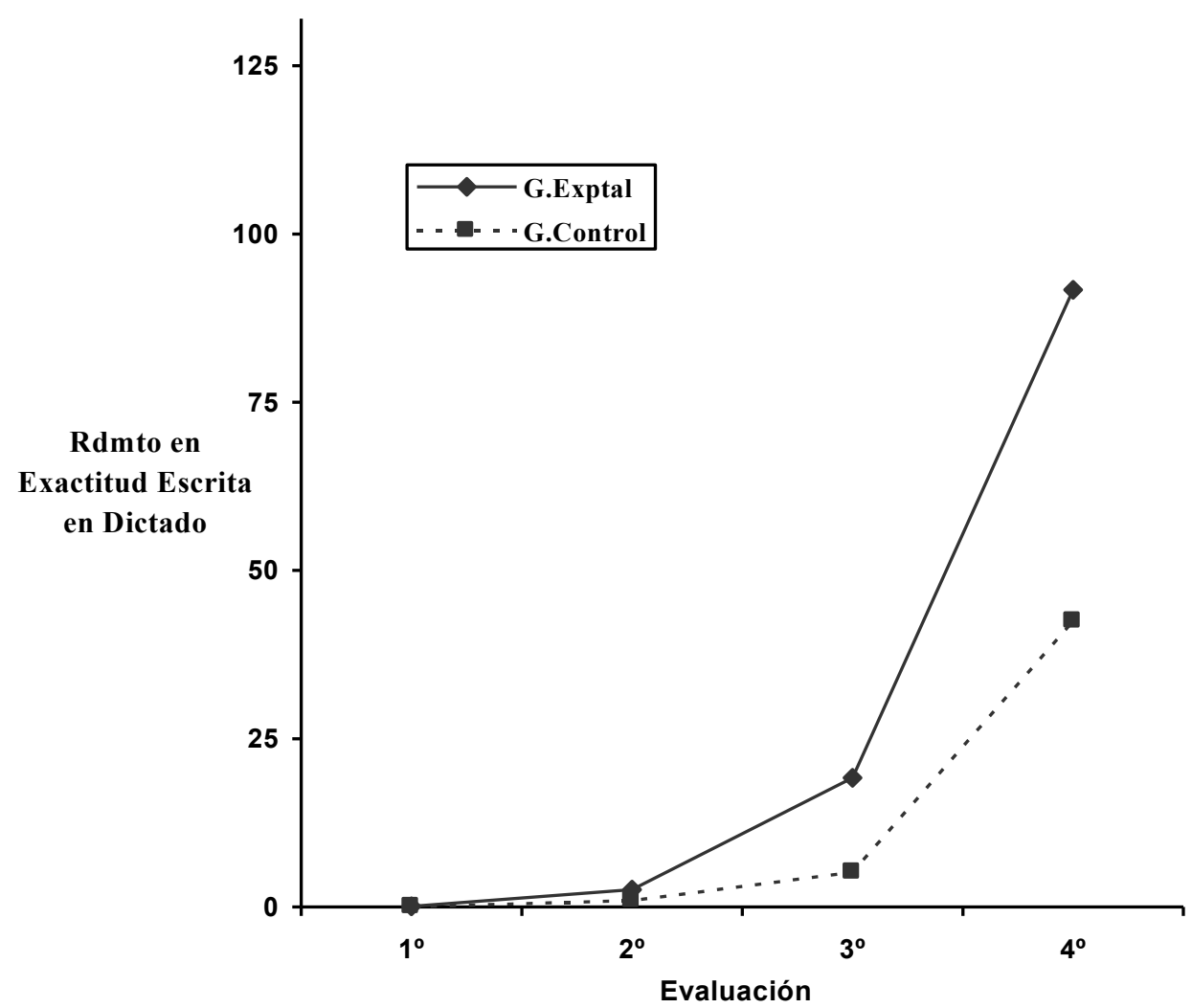

Figura 2. Puntuaciones medias obtenidas en el Rendimiento en Exactitud en Dictado.

Para analizar si estas diferencias son significativas y debidas al efecto de la intervención se llevó a cabo el análisis de varianza de medidas repetidas en esta variable de estudio, comprobándose el supuesto de Homocedasticidad $\left(F_{(1,104)}==2.32\right.$ y p $\left.=0.13\right)$ y verificándose el supuesto de Esfericidad $(\varepsilon=0.50)$.

El análisis de varianza de medidas repetidas muestra (Tabla 3) que existen diferencias estadísticamente significativas entre los grupos control y experimental, $\left(F_{(1,104)}=70.73\right.$; $\mathrm{p}<0.001)$. Se rechazaría la hipótesis nula del factor inter-sujeto referida a que no existen diferencias entre las medias de los grupos en rendimiento en exactitud en dictado. Así mismo, hay que resaltar en este factor la alta adecuación del contraste de hipótesis, obteniéndose una excelente potencia $(\beta=1)$ y un considerado tamaño del efecto, con $\eta^{2}=0.40$ (Tabla 3 ). 
Por otro lado, los resultados rechazan la hipótesis nula del factor intra-sujetos, referente a la no existencia de diferencias significativas entre las diversas evaluaciones, ya que se muestra un cambio significativo a lo largo de las cuatro evaluaciones $\left(F_{(1 ' 52,158,42)}=403.55\right.$; $\mathrm{p}<0.001)$. Nuevamente, se encuentra una excelente potencia de contraste de hipótesis $(\beta=1)$ en ambos grupos y un considerado tamaño del efecto $\left(\eta^{2}=0.79\right)$ (Tabla 3$)$.

Así mismo, se encuentra un efecto significativo de interacción evaluación y grupo $\left(F_{\left(1^{\prime} 52,158,42\right)}=52.58 ; \mathrm{p}<0.001\right)$, rechazando así la hipótesis que hace referencia a la no existencia de diferencias significativas entre el grupo experimental y el grupo control en las diversas evaluaciones. Se obtiene una máxima potencia de $\beta=1$ y un considerado tamaño del efecto de $\eta^{2}=0.33$, como se puede observar en la tabla 4 . Es decir, se han encontrado diferencias significativas entre el grupo experimental y control a lo largo de las diferentes evaluaciones, a favor del grupo experimental, debido a la aplicación del programa de intervención.

Los contrastes de medida intra-sujeto, muestran significativo el efecto lineal, cuadrático y cúbico para las evaluaciones $\left(F_{(1,104)}=504.08,356.57\right.$ y $61.98 ; \mathrm{p}<0.001$, respectivamente $)$ y el efecto lineal y cuadrático también resulta significativo para la interacción $\left(F_{(1,104)}=72.04\right.$ y 35.54; $\mathrm{p}<0.001$ ). Por tanto, puede concluirse que el modelo más plausible para explicar la variabilidad del Rendimiento en Exactitud en Dictado, es el cuadrático, recogiendo los efectos de la evaluación y de su interacción con el grupo.

Por otra parte, tras examinar las diferencias post-hoc mediante el método de Bonferroni, para los niveles de interacción entre cada una de las evaluaciones en cada uno de los grupos, los resultados encuentran que en el grupo experimental existen diferencias estadísticamente significativas entre las diferentes evaluaciones, mientras que en el grupo control, las diferencias son significativas únicamente entre la primera, segunda y tercera con la cuarta evaluación (Tabla 4). Es decir, se encuentran diferencias significativas en el grupo experimental entre los cuatro y los cinco años, entre los cuatro y los seis años y entre los cinco y los seis; sin embargo, en el grupo control estas diferencias solo se observan a partir de los seis años (escolaridad obligatoria) con respecto a las puntuaciones obtenidas por estos niños a los cuatro y cinco años. 
Por último, las diferencias post-hoc encontradas con la corrección de Bonferroni para los niveles de interacción entre cada grupo en cada evaluación, nos muestran que existen diferencias estadísticamente significativas en la interacción entre ambos grupos en cada evaluación, excepto en la evaluación inicial (pretest) donde no se han encontrado diferencias significativas entre los dos grupos (Tabla 5). Es decir, se encontraron diferencias estadísticamente significativas entre ambos grupos a partir de la segunda evaluación, a favor del grupo experimental, siendo esta diferencia mayor a medida que se avanza en los años de aplicación del programa. De esta manera, como se puede comprobar en la tabla 5 , el número de periodos de intervención que han recibido los sujetos, así como el grupo al que pertenecen, explican las diferencias observadas en el rendimiento en exactitud en dictado. Es decir, que no sólo se encuentra que el grupo experimental obtiene un mejor rendimiento en exactitud en dictado que el grupo control, sino que, además, las diferencias encontradas entre ambos grupos en dicho rendimiento, aumentan a medida que se avanza en los periodos de intervención que reciben los sujetos del primer grupo (GE).

Por tanto, podemos concluir, que los dos grupos avanzan en el transcurso de los diferentes niveles educativos, pero que el grupo al que se le aplicó el programa de intervención psicoeducativa en el lenguaje escrito ha obtenido un mayor Rendimiento en Exactitud en Dictado que el grupo al que no se le aplicó; y que, además, estas diferencias en Rendimiento en Exactitud en Dictado es mayor cuanto mayor es el nivel educativo, debido a la aplicación del programa de intervención.

\section{Conclusiones}

En este trabajo se ha intentado comprobar la mejora del proceso de enseñanzaaprendizaje de la escritura, en términos de copia y dictado, debida a la aplicación de un programa de intervención psicoeducativa desde Educación Infantil hasta finalizar el primer curso de Educación Primaria.

Los resultados muestran la existencia de diferencias significativas en el rendimiento en copia y dictado en los factores inter-sujeto, intra-sujeto y en la interacción grupo y eva- 
luación, destacándose una alta potencia y un considerado tamaño del efecto de la intervención en cada una de las variables. Es decir, que aunque los dos grupos avanzan en el transcurso de los diferentes niveles educativos, se han encontrado diferencias estadísticamente significativas entre los grupos experimental y control en las distintas evaluaciones, a favor del grupo experimental, debido a la aplicación del programa de intervención psicoeducativa.

En cuanto a los análisis a posteriori realizados estos desvelan, por un lado, que en el rendimiento en copia se han encontrado diferencias entre todas las evaluaciones en ambos grupos, es decir, que ambos grupos han avanzado significativamente a lo largo de los años en esta variable. Esto puede ser debido a que el curriculum oficial establecido para Educación Infantil fomenta también la copia, de ahí que sus puntuaciones en esta variable avancen en los diferentes niveles educativos al igual que los sujetos del grupo experimental. Sin embargo, por otro lado, en rendimiento en dictado, las diferencias se hacen más patentes y significativas solo en el grupo experimental, desde el primer año de aplicación del programa; es decir, desde los cuatro años, encontrándose diferencias entre los cuatro y los cinco años, entre los cinco y los seis años y entre los cuatro y los seis años. Por el contrario, los cambios encontrados en el grupo control en esta variable son más perceptibles y patentes en esta variable únicamente a partir de los seis años, cuando los niños entran en la educación primaria y comienzan con la enseñanza formal de la lectoescritura, siendo entonces los objetivos curriculares oficiales más específicos.

Cabe destacar, también, que las diferencias entre los grupos se mantienen o crecen en rendimiento en copia y dictado, respectivamente, a medida que se avanza en los años de aplicación del programa. Además, cuando terminan el primer curso de Educación primaria el grupo al que se le aplica el programa alcanza mejores resultados, tanto en copia como en dictado, que aquellos que no recibieron la intervención psicoeducativa programada.

En definitiva, se puede concluir que no sólo el grupo al que se le aplicó el programa de intervención psicoeducativa ha obtenido un mayor rendimiento en escritura, en términos de copia y dictado, sino que, además, el rendimiento es mayor cuanto más periodos de intervención hayan recibido los niños. Se concluye, pues, que los avances en rendimiento en escritura (copia y dictado) de los niños a los que les ha sido aplicado el programa han sido producidos 
por la aplicación del mismo. A este respecto, hay que destacar la importancia de la preparación de las maestras y de la estructuración, sistematización y naturaleza de los contenidos del programa de intervención psicoeducativa que ha sido diseñado y aplicado desde edades tempranas, ya que estas características han contribuido a alcanzar estos resultados.

Desde la investigación educativa, estos resultados confirman nuevamente los encontrados en otras lenguas y otros sistemas educativos que abogan por la enseñanza de la lectura y la escritura desde temprana edad, proponiendo cambios curriculares en los proyectos de centros (Allington y Woodside-Jiron, 1999; Baroccio, 1997; Burns, et al. 1999; Escamilla, 1994; Osborn y Lehr, 1998; Russo, et al. 1998; Slavin, 1995; Swartz, 1996). Por tanto, aunque en el currículum escolar oficial se propone en edades tempranas un acercamiento al lenguaje escrito a partir de la enseñanza del lenguaje oral, dicha enseñanza tendría que llevarse a cabo de manera sistemática, constante y específica.

Los resultados demuestran también que los niños a estas edades pueden aprender a escribir desde edades tempranas correctamente si reciben una enseñanza adecuada y si se les prepara conscientemente, trabajando y entrenándoles en todas aquellas variables cognitivolingüísticas que fomentan el desarrollo del lenguaje escrito tales como el conocimiento fonológico, el desarrollo fonológico, el desarrollo semántico, y el desarrollo morfosintáctico (Calero y Pérez, 1993; Carrillo, 1994; González, 1993; Jiménez, 1992; Muter y Snowling, 1998; Slavin, 1995; Stanovich, 1992; Swartz, et al. 2000). Además, se destaca la importancia de priorizar así el proceso de enseñanza-aprendizaje de la escritura, creándoles un hábito lectoescritor a través de la sistematización de distintas actividades lectoescritoras y aumentando el tiempo de exposición a ellas (Baroccio y Haggs, 1999; Button, et al. 1996; Pinnell y McCarrier, 1994; Slavin, 1995; Slavin, Madden, Dolan y Wasik, 1996; Stanovich, 1994; Swartz, et al. 2000). En Australia (1984), Canadá (1988), California (1991), Gran Bretaña (1991) y México (1997), como consecuencia del problema del bilingüismo y de los problemas de alfabetización que están encontrando en las últimas décadas, se viene desarrollando la aplicación de varios proyectos de investigación en esta línea (Allington y Woodside-Jiron, 1999; House, 1996; Jones, 1996).

Finalmente, cabe destacar que en trabajos posteriores, se pretende comprobar si la aplicación de este programa de intervención consigue aminorar la aparición de las dificultades Revista Electrónica de Investigación Psicoeducativa, No 13 Vol 5 (3), 2007. ISSN:1696-2095. pp:651-678 
de aprendizaje y si mejora también el rendimiento académico de los escolares. También seria interesante poder comprobar si transcurrido algunos años de la aplicación del programa, las diferencias en el rendimiento en escritura siguen manteniéndose entre los grupos, ya que si esto ocurriese la importancia y ventajas de la intervención temprana en el lenguaje escrito sería aún más patente, como ocurre en otros sistemas educativos y otras lenguas.

\section{Agradecimientos}

Este trabajo forma parte del Proyecto de Investigación BSO 20011826, financiado por el MCyT y con fondos Feder.

\section{Referencias}

Adams, M. (1998): Phonemic awareness in young children. Baltimore, Paul H. Brookes Publishing Co.

Allington, R.L., \& Woodside-Jiron, A.D. (1999). The politics of literacy teaching: How research shaped educational policy. Educational Researcher, 28 (8).

Anguera, M.T., Arnau, J., Ato, M., Martínez, R., Pascual, J., y Vallejo, G. (1995). Métodos en investigación en Psicología. Barcelona: Síntesis.

Baroccio, R. (1997). California early literacy learning and the problem of school change. En Swartz, S. (Ed). Processing of the international congress on challenges to education. San Bernardino, CA: California State University.

Baroccio R. \& Hagg, C. (1999). Enseñanza Inicial de la lectoescritura. México: Universidad de México.

Blair-Larsen, S.M. \& Williams, K.A. (1999). The balanced reading program. Newark, USA: International reading Association.

Blanca, M.J. y Rando, B. (1999). Ejercicios de diseños experimentales básicos en Psicología. Málaga: Servicio de Publicaciones de la Universidad.

Burns, M.S., Griffin. \& Snows, C.E. (1999). Starting out rigth. A guide promoting children's reading success. WDC, USA: National Academic Press.

Button, K., Johnson, M.J. \& Ferguson, P. (1996). Interactive writting in a primary classroom. The reading teacher, 49 (6), 446-454. 
Calero, A. y Pérez, R. (1993). Segmentación del habla y adquisición de la lectura. Comunicación, Lenguaje y Educación, 18, 41-53.

Condemarín, M.; Chadwick, M.; Milicia, N. (1985): Madurez escolar. Madrid, CEPE.

Carrillo, M. (1994). Development of phonological awareness and reading acquisition. A study in Spanish language. Reading and Writing: An Interdisciplinary Journal, 3 (6), 279298.

Casalis, S. \& Louis-Alexandre, M.F. (2000). Morphological analysis, phonological analysis and learning to read French: a longitudinal study. Reading and Writing: An Interdisciplinary Journal, 12, 303-335.

Da Fontoura, H. A. \& Siegel, L.S. (1995). Reading, syntactic, and working memory skills of bilingual Portuguese- English Canadian Children. Reading and Writing: An Interdisciplinary Journal, 7, 139- 153.

Decreto 105/1992, de 9 de Junio por el que se establecen las enseñanzas correspondientes a la Educación Primaria. Boletín Oficial de la Junta de Andalucía 56/92, 20 de junio de 1992.

Decreto 107/1992, de 9 de Junio por el que se establecen las enseñanzas correspondientes a la Educación Infantil en Andalucía. Boletín Oficial de la Junta de Andalucía 56/9, 20 de junio de 1992.

Defior, S. y Tudela, P. (1994). Effect of phonological training on reading and writing acquisition. Reading and Writing: An Interdisciplinary Journal, 3 (6), 299-320.

Delgado, M., Martín, I., Rivas, T. y González, M.J. (2004). Formación de educadores y mejora del proceso de enseñanza-aprendizaje de la escritura en Educación Infantil. En M.I. Fajardo, M.I. Ruiz, A.V. Dáz y F. Vicente (Eds). Infancia y Adolescencia: Desarrollo psicológico y propuestas de intervención (pp 149-157). Badajoz: Psicoex.

Downing, J.; Thackray D.V.(1974): Madurez para la lectura. Buenos Aires, Kapelusz.

Elbro, C. (1996): "Early linguistic abilities and reading development: A review and a hypothesis", Reading and Writing: An Interdisciplinary Journal, 8, 453-485.

Escamilla, K. (1994). Descubriendo la lectura: un programa en español de lectoescritura para las primeras etapas. An International Journal of Early Literacy, 1 (1), 71-85.

Fountas, J. y Pinnell, G.S. (1996). Guided reading. Pontsmonth, N H: Heinemann.

Gillon, G. y Dodd, B.J.(1994) A prospective study of the relationship between phonological, semantic and syntactic skills and specific disability. Reading and Writing: An Interdisciplinary Journal, 6, 321-345. 
González, M.J. (1993). Estudio evolutivo del aprendizaje de la lectura: análisis causal de la influencia de variables de desarrollo fonológico y psicolingüístico y de variables contextuales con niños, normales y con dificultades de aprendizaje. Málaga, España: SPICUM, Universidad de Málaga.

González, M.J. (1996). Aprendizaje de la lectura y conocimiento fonológico: Análisis evolutivo e implicaciones educativas. Infancia y Aprendizaje, 76, 97-107.

House, E.R. (1996). A frameword for appraising educational reforms. Educational researcher, vol 25, (7), 35-46.

Jiménez, J.E. (1992). Metaconocimiento fonológico: estudio descriptivo sobre una muestra de niños prelectores en edad preescolar. Infancia y Aprendizaje, 57, 49-66.

Jiménez, J. y Artiles, C. (1990). Factores predictivos del éxito en el aprendizaje de la lectoescritura. Infancia y Aprendizaje, 49, 21-36.

Jones, L.V. (1996). A history of the national assessment of educational progress and some questions about its future. Educational Researcher, vol 25 (7), 214-222.

Mahony, D.; Singson, M.; Mann, V. (2000): "Reading ability and sensitivity to morphological relations", Reading and Writing: An Interdisciplinary Journal, 12, pp. 219- 252.

Molina, S. (1991): Batería diagnóstica para la competencia básica para el aprendizaje de la lectura. Madrid, CEPE.

Muchelli, B.; Bourcier, J. (1985): La dislexia. Causas, diagnóstico y reeducación. Madrid, Cincel.

Muter, V. \& Snowling, M. (1998). Concurrent and longitudinal predictors of reading: the role of metalinguistic and short-term memory skills.Reading Research Quartely, 33 (3), 231-242.

Osborn, J. \& Lehr, L. (1998). Literacy for all. The Guilford issues in teaching and learning. New York, USA: Academic Press.

Pinnell, J.S. \& Mc Carrier, D. (1994). Interactive writing: A transition tool for assisting children in learning to read and write. In E. Hiebert y L. B. Taylor (Eds.). Getting reading right from the start: Effective early literacy interventions. Needham Heights, MA: Allyn y Bacon.

Russo, M., Kosman, G., Ginsburg, A., Thompson-Hoffman, S. \& Pederson, J. (1998). A compact for reading and School-home Links. USA : Department of Education. 
Revuelta, R.; Guillén, F. (1987): Madurez y edad inicial para la lectura. Comunicación presentada al Quinto C.E.L, 154-155.

Seymor, P. H. \& Evans H.M. (1994). Levels of phonological awareness and learning to read. Reading and Writing: An Interdisciplinary Journal, 3 (6), 221-250.

Slavin, R. E. (1995). Success for all: a summary of research. Annual meeting of American educational research association. San Francisco, California: University of California.

Slavin, R. E., Madden, N. A., Dolan, L. y Wasik, B. A. (1996). Every child, every school: Success for all. California: Corwin Press, Inc.

So, D. y Siegel, L.S.(1997) Learning to read Chinese: Semantic, syntactic, phonological and working memory skills in normally achieving and poor Chinese readers. Reading and Writing: An Interdisciplinary Journal, 9, 1-21.

Stanovich, K.E. (1992). Speculations on the Causes and consequences of individual differences on Early reading acquisition. En PH.B. Gough, L.C. Ehri y R. Treiman. Reading Acquisition. Hillsdale: Lawrence Erlbaum Associate.

Stanovich, K.E. (1994). Constructivism in reading education. The Journal of Special Education, $28(3), 312-322$.

Swartz, E. (1996). Teaching Reading. Reading Program Advisory. CA: California Department of Education.

Swartz, S.L., Shook, R.E. y Klein, A.F. (2000). California Early Literacy Learning. Redlaus, CA: Foundation for California early literacy learning. 
María José González-Valenzuela et al.

[Página en blanco por razones de paginación] 